\title{
RESEARCH
}

Open Access

\section{High-speed visible light communication systems based on Si-substrate LEDs with multiple superlattice interlayers}

Fangchen $\mathrm{Hu}^{1}$, Shouqing Chen ${ }^{2}$, Yuyi Zhang ${ }^{3}$, Guoqiang Li ${ }^{1}$, Peng Zou ${ }^{1}$, Junwen Zhang ${ }^{1}$, Chao Shen ${ }^{1}$, Xiaolei Zhang ${ }^{3}$, Jian $\mathrm{Hu}^{2}$, Jianli Zhang ${ }^{2}$, Zhixue $\mathrm{He}^{4,5}$, Shaohua $\mathrm{Yu}^{4}$, Fengyi Jiang ${ }^{2}$ and Nan $\mathrm{Chi}^{{ }^{*}}$

* Correspondence: nanchi@fudan. edu.cn

${ }^{1}$ Key Laboratory for Information Science of Electromagnetic Waves (MoE), Fudan University, Shanghai 200433, People's Republic of China Full list of author information is available at the end of the article

\begin{abstract}
High-speed visible light communication (VLC), as a cutting-edge supplementary solution in $6 \mathrm{G}$ to traditional radio-frequency communication, is expected to address the tension between continuously increased demand of capacity and currently limited supply of radio-frequency spectrum resource. The main driver behind the high-speed VLC is the presence of light emitting diode (LED) which not only offers energy-efficient lighting, but also provides a cost-efficient alternative to the VLC transmitter with superior modulation potential. Particularly, the InGaN/GaN LED grown on Si substrate is a promising VLC transmitter to simultaneously realize effective communication and illumination by virtue of beyond 10-Gbps communication capacity and Watt-level output optical power. In previous parameter optimization of Si-substrate LED, the superlattice interlayer (SL), especially its period number, is reported to be the key factor to improve the lighting performance by enhancing the wall-plug efficiency, but few efforts were made to investigate the influence of SLs on VLC performance. Therefore, to optimize the VLC performance of Si-substrate LEDs, we for the first time investigated the impact of the SL period number on VLC system through experiments and theoretical derivation. The results show that more SL period number is related to higher signal-to-noise ratio (SNR) via improving the wall-plug efficiency. In addition, by using Levin-Campello bit and power loading technology, we achieved a record-breaking data rate of 3.37 Gbps over 1.2-m free-space VLC link under given optimal SL period number, which, to the best of our knowledge, is the highest data rate for a Si-substrate LED-based VLC system.
\end{abstract}

Keywords: Visible light communication, InGaN/GaN LEDs design, Silicon photonics, Superlattice interlayers, 6G
๑ The Author(s). 2021 Open Access This article is licensed under a Creative Commons Attribution 4.0 International License, which permits use, sharing, adaptation, distribution and reproduction in any medium or format, as long as you give appropriate credit to the original author(s) and the source, provide a link to the Creative Commons licence, and indicate if changes were made. The images or other third party material in this article are included in the article's Creative Commons licence, unless indicated otherwise in a credit line to the material. If material is not included in the article's Creative Commons licence and your intended use is not permitted by statutory regulation or exceeds the permitted use, you will need to obtain permission directly from the copyright holder. To view a copy of this licence, visit http://creativecommons.org/licenses/by/4.0/. 


\section{Introduction}

As the emergence of the innovative technologies requiring for ultra-high communication data rate (such as artificial intelligence, virtual reality and the Internet of Things), the wireless communication research focus has gradually moved to 6G, which is likely to offer a two orders of magnitude bit-rate growth over 5G [1]. The current wireless communication network suffers from shortage of radio-frequency (RF) spectrum resources, hardly to reach giga-bit-per-second data rate. Hence, visible light communication (VLC) is rapidly emerging as a promising supplementary technology for RF communication [2-4], which can provide wide unlicensed spectrum resource (ranging from 400 to $800 \mathrm{THz}$ ) to alleviate the "Wi-Fi spectrum crunch" [5] issue. Additionally, using light-emitting-diode (LED) as VLC transmitter dramatically saves the cost compared to that of laser-based system, and thus making LED-based VLC system a popular and accessible wireless communication configuration. Currently, the LED-based VLC system has reached the data rate of Mega-bit-per-second using off-the-shelf LEDs [6], but these LEDs are originally designed for daily lighting where their bandwidth is only several Mega-Hertz (MHz) [7]. If the LED is well designed (e.g. reducing LED area), the achievable data rate could reach Giga-bit-per-second level [8]. That's to say, novel LED design or parameter optimization is critical to further increase data rate. Specifically, the InGaN/GaN vertical LED grown on silicon $(\mathrm{Si})$ substrate, known as Si-substrate LED, is a promising alternative to high-speed VLC transmitter, which could not only support over 10-Gbps VLC underwater link [9], but also offer effective white-light illumination thanks to its Watt-level output optical power and the competitive external quantum efficiency within all visible light spectrum.

Before the Si-substrate LED is deeply investigated, due to the lack of large-size GaN bulk substrate with structural perfection, the commercial InGaN-based LEDs are normally grown on sapphire and $\mathrm{SiC}$ substrates. Sapphire is currently the most commonly used substrate for commercial LED fabrication, but it still imposes constraint on the GaN film quality due to its poor thermal conductivity, relatively high thermalexpansion coefficient mismatch (7.5\%) and lattice mismatch (15.7\%) to GaN [10]. When high current is injected into the LED grown on sapphire substrate, sapphire prevents the efficient dissipation of heat generated by the non-radiative recombination process in LEDs. Excess heat accumulation would significantly inhibit the LED performance, causing undesired droop effect and light efficiency decrease. In addition, the LED on a sapphire substrate requires all contacts must be placed on the top side owning to the insulating property of sapphire if the GaN film is not lifted off from the sapphire substrate. Such a lateral structure complicates the contact and package schemes. The operation voltage is also increased because of the induced serious current crowding and current droop effect under this LED configuration. For the $\mathrm{SiC}$ substrate, despite the merits of good thermal and electrical conductivity, and less lattice mismatch to GaN (only 3.5\%) than sapphire [11], the high cost restrains its wide application. Consequently, the limitation motivates the development of the Si substrate for LEDs growth. It not only has a lower cost, a larger size, the good thermal and electrical conductivity [12], but also easily realizes the vertical structure by a simple wet chemical etching technology to lift off the GaN film from the Si substrate. In contrast, the sapphiresubstrate LED needs laser lift-off technology, which will inevitably damage the crystal quality [13]. Moreover, the external quantum efficiency (EQE) of Si-substrate LEDs was found to be higher than that of sapphire-substrate LEDs at the injection current $>300$ $\mathrm{mA}$ [14]. Nevertheless, there is a large lattice mismatch in the lattice constants (17.1\%) 
and the thermal expansion coefficients (54\%) between $\mathrm{Si}$ and $\mathrm{GaN}$, respectively resulting in the high dislocation density and the generation of cracks and bend of GaN films. To overcome these problems, plenty of innovative optimization methods were proposed to modify the structure of Si-substrate LEDs, such as the low-temperature AlN: $\mathrm{Si}$ interlayers technology [15], the step-graded AlGaN intermediate layers technology [16] and patterned Si substrate selective-area-growth technology [17, 18], etc. These technologies markedly increased the EQE of Si-substrate LEDs, especially for green and yellow LEDs. The wall-plug efficiency (WPE) of 565-nm yellow Si-substrate LEDs has reached $24.3 \%$ at the current density of $20 \mathrm{~A} / \mathrm{cm}^{2}$, which is the highest WPE of yellow LED to the best of knowledge.

However, these optimization investigations for high-efficient Si-substrate LED is primarily driven by the application of solid-state lighting rather than high-speed VLC, few efforts were made to enhance VLC performance, which requires the LED to possess wide bandwidth and high modulation efficiency from the perspective of communication, from direct Si-substrate LED parameters optimization. The high modulation efficiency means the LED could provide a high SNR for the modulated optical signal after the signal intensity modulation. Nevertheless, the specific requirement in the device level is still unclear. For LED's lighting functionality, the superlattice interlayer (SL) is a key stress-relief layer in the epitaxy-structure of Si-substrate LEDs to improve the lighting performance [19]. It consists of dozens of periods of InGaN well layers and GaN barrier layers. The value of the periodicity of the InGaN/GaN structure is defined the SL period number which greatly affects the LED's EQE and forward voltage [20], but its impact on VLC performance is unsure. The enhancement of lighting performance for a LED does not directly indicate the improvement of VLC performance. Therefore, we for the first time analyzed the impact of the SL period number on VLC performance based on three LED samples with different SL period numbers. We found that increasing SLs period number substantially enhances the bandwidth and modulation efficiency by reducing the carrier lifetime and improving the WPE of LED, respectively. We also theoretically derived that the modulation efficiency depends on the WPE according to the knowledge of semi-conductor physics. This conclusion not only helps LED designer make a qualitative judgement on the modulation efficiency of a LED directly from the device level, but also accelerates the development of future Si-substrate LED design to achieve a higher VLC capacity through SNR enhancement. Based on a blue Si-substrate LED with optimal SL period number, we realized a record-breaking data rate of 3.37 Gbps over a $1.2 \mathrm{~m}$ free-space link using the bit and power loading discrete multi-tone (BPL-DMT) modulation. If the transmission distance extends to $3 \mathrm{~m}$, the data rate can still reach up to 3 Gbps. These results demonstrate Si-substrate LEDs could realize both the effective illumination function using a large light-emitting area (45*45 mil) and a high-speed data transmission over a regular indoor access distance (within $3 \mathrm{~m}$ ).

\section{Methods}

Fabrication and design of the vertical-structure Si-substrate LED with multiple SLs

To investigate the impact of different SL period number on VLC performance, three Si-substrate LED samples with the SL period number of 8, 15, 32 are first prepared, labeled as Sample A, Sample B and Sample C, respectively. Figure 1 (a) depicted the 


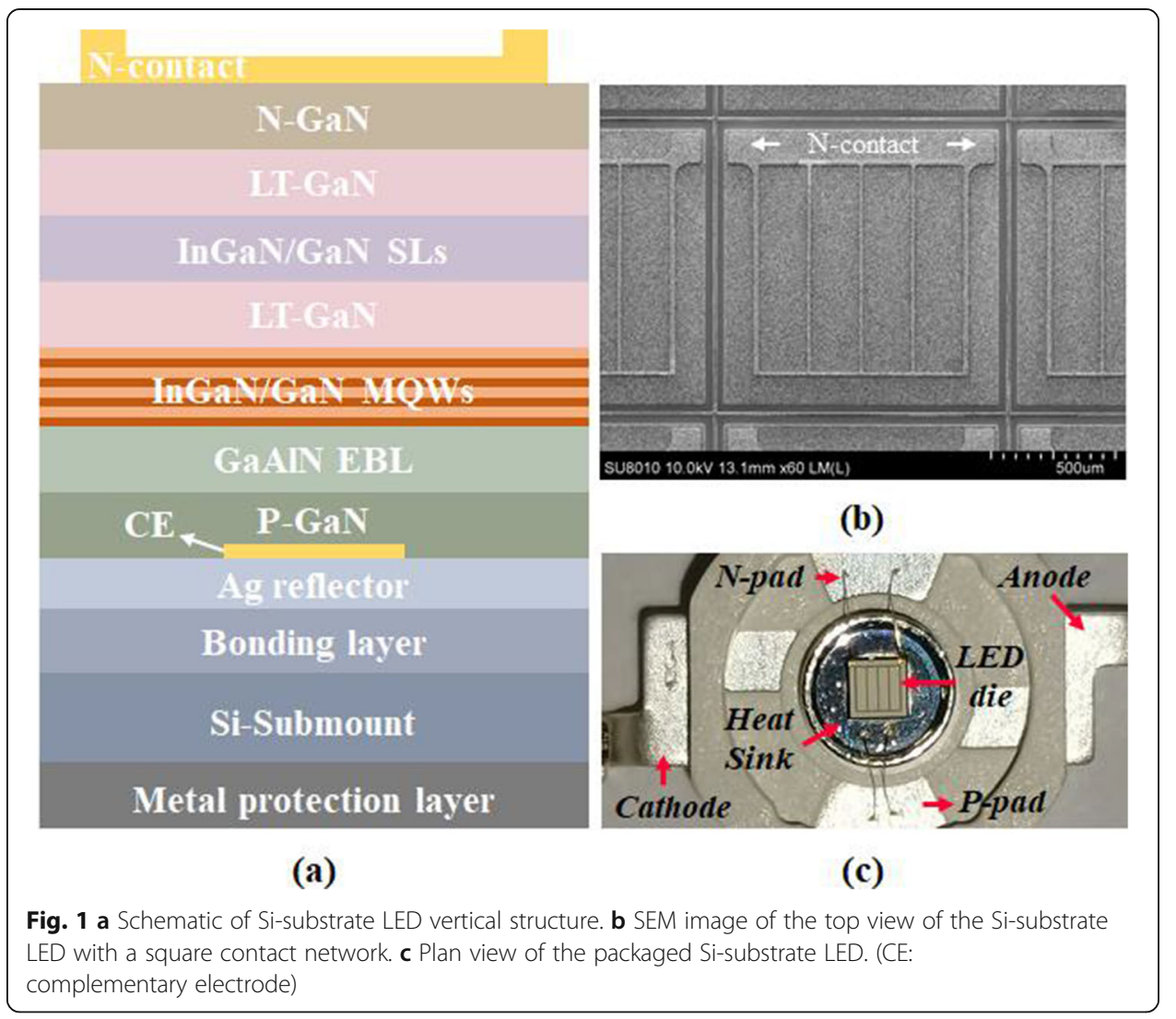

schematic of the epitaxial and vertical structure of LED samples. Initially, the epitaxial wafers of the three LED samples are fabricated by the close-coupled showerhead metalorganic chemical vapor deposition (CCS-MOCVD). According to the standard epitaxial growth process of Si-substrate LED [21], the AlN buffer layer is first deposited on the patterned Si (111) substrate to relieve the large lattice mismatch between Si and GaN, then followed by a Si-doped N-type GaN layer, a 10-nm low-temperature GaN (LT$\mathrm{GaN}$ ) layer, an $\mathrm{In}_{0.15} \mathrm{Ga}_{0.85} \mathrm{~N} / \mathrm{GaN}$ SL layer and another LT-GaN layer. These layers all serve as significant strain relaxation layers beneath the InGaN/GaN multiple quantum wells (MQWs) to partially reduce the quantum-confined Stark effect (QCSE) [22]. Above the MQWs layer, a Mg-doped p-Al0.2Ga0.8n electron blocking layer (EBL) and a Mg-doped p-GaN layer are deposited in order. Once the epitaxial growth is completed, the Si substrate and the AlN buffer layer are removed by wet etching technology, and the rest of film is inverted with the N-GaN layer upwards. An Ag reflector layer is subsequently deposited on the P-GaN to serve as a p-Ohmic contact and a high-reflectivity reflector. The EQE of Si-substrate LED is greatly improved with the property of good unidirectional light emitting benefitted from the Ag reflector layer and an embedded complementary electrode technology [23]. Next, the $\mathrm{Cr} / \mathrm{Pt} / \mathrm{Au}$ are deposited on the roughed $\mathrm{N}-\mathrm{GaN}$ to form a square N-contact network shown in the scanning electron microscopy (SEM) image (Fig. 1b). This electrode network could improve the uniformity of the emitted light intensity. To protect and support LED, a bounding layer, a Si-submount and a metal protection layer are stacked under the $\mathrm{Ag}$ reflector layer. The detailed fabrication of vertical-structure Si-substrate LED is 
reported in Ref. $[9,12]$. Figure.1c) is the plan view of the packaged Si-substrate LED with side length of 45 mil. The LED die is directly attached to the heat sink of the bracket by a high thermal-conductive and adhesive CT285 silver paste. Two groups of golden wire (diameter:20um) electronically connect LED die and the bracket. One group connects P-contact (Ag reflector) of the LED die to the P-pad of the bracket as the anode, the other connects the N-contact to the N-pad as the cathode. The optoelectronic characteristics of the three LED samples were measured by a Keithley Instruments 2635A source meter and an Instrument Systems CAS 140CT spectrometer.

\section{Experimental setup of VLC system and principle of BPL-DMT modulation}

To evaluate the VLC performance of the Si-substrate LED with different SL period number, a VLC experiment setup is established as the illustration of Fig. 2a). A 16 GSa/s arbitrary waveform generator (AWG, Keysight M8190A) is used to generate an analog signal stream. A constant-resistance symmetrical bridged-T amplitude preequalizer (Pre-Eq.) is followed to expand the bandwidth of the transmitter [24]. After amplified by an electronic amplifier (EA, mini circuits ZHL-6A-S+), the signal is coupled with the direct current (DC) by a bias tee (mini circuits ZFBT-4R2GW-FT+) to drive the lighting of the LED sample. Two plano-convex lenses placed in front of the LED and one plano-convex lens are used to guide the emitted light onto the PINphotodetector (PIN-PD, HAMAMATSU S10784). To prevent the saturation effect of PIN-PD, an adjustable neutral-density filter (ND filter) is placed between the PIN-PD and the lens at the receiver side. Subsequently, the converted electrical signal outputted from the PIN-PD is amplified by a trans-impedance amplifier (TIA). Its two differential outputs are connected to two EAs for the second-class amplification for a minimum of the quantization error of the oscilloscope (OSC, Agilent MSO9054A), which is followed at the end of the receiver. The utilized devices and system configuration are shown in Fig. 2b-d).

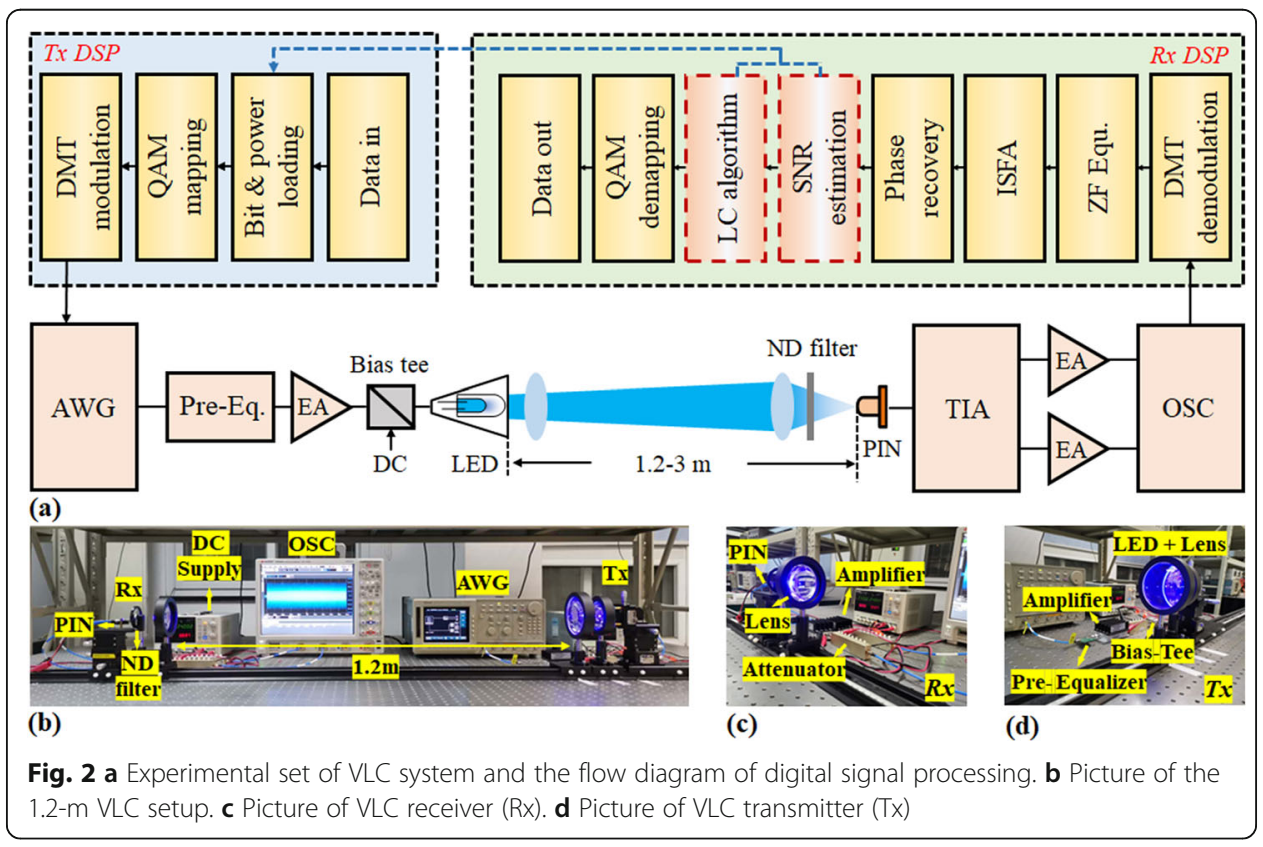


The principle of BPL-DMT modulation is shown in the upper flow diagram of Fig. 2a). First, system SNR should be measured to guide the optimal bit allocation. To maximize the measured SNR, the optical power of the light onto the PIN-PD needs to approach the threshold optical power of PIN-PD to get the maximum PD response, and thus the attenuation value of the ND filter needs adaptive adjustment. The training signal for SNR measurement is a DMT-modulated quadrature phase shift keying (QPSK) signal generated according to the standard DMT modulation process [25]. After it is transmitted over the VLC channel, the received signal from OSC is immediately demodulated into a QPSK signal referred to standard DMT demodulation process, which is subsequently equalized by classical zero-forcing equalization and intra-symbol frequency-domain averaging (ISFA). Next, the SNR could be estimated by calculating the error vector magnitude between the transmitted QPSK and the received QPSK signal [26]. The forward error code (FEC) threshold uses the 7\% overhead FEC threshold with the bit error ratio (BER) of 3.8e-3. The ISFA technology is applied to smooth the estimated SNR response and suppress noise.

Once the SNR is obtained, it can be loaded into the Levin-Campello (LC) algorithm to automatically generate a suitable bit number and power ratio allocation strategy for every subcarrier $[27,28]$. The loaded bit number in total could be maximized based on the allocation strategy. The value of spectral efficiency (SE) is just the average value of bit numbers in every subcarrier. Subsequently, the signal flow is mapped to quadrature amplitude modulation (QAM) signal accordingly to the allocation strategy and the QAM signal is modulated to a DMT signal, which is fed into the AWG. At the receiver, the received signal is resampled and synchronized at first, then demodulated to a QAM signal followed by ZF equalization and ISFA. Due to the clock mismatching between AWG and OSC, the frequency and phase offset need to be compensated. The pilot estimation method [29] is utilized to recover the offset. After the phase recovery, the QAM de-mapping is followed to transfer the received signal to a decimal signal sequence, so the BER can be calculated. If the BER is lower than $3.8 \mathrm{e}-3$, the achieved data rate can be obtained by the multiplication of SE and modulation bandwidth.

\section{Measurement methods of S21 parameters and - 10-dB bandwidth}

The S21 parameters are measured also based on the experimental setup in Fig. 2a), but the AWG and OSC should be replaced by a network analyzer (Agilent Technologies, N5230C). The network analyzer has two ports, one outputs the swept-frequency signal, and the other receives the received signal which has been transmitted over the whole VLC system. The S21 parameter could accurately reflect the frequency response. The corresponding $-10-\mathrm{dB}$ bandwidth of the system could also be read from the smoothened S21 curve. When injection current increases, the saturation effect of PD should be prevented, and thus the attenuation of the ND filter is set to a relatively big value that there is no saturation effect at the current of $300 \mathrm{~mA}$. In addition, the power of sweptfrequency signal is set to $-10-\mathrm{dBm}$ to guarantee the measured bandwidth is under a small-signal case.

Measurement methods of the time-domain transient response of LEDs

Based on the setup in Fig. 2a), the time-domain transient response of the system can be measured by transmitting a Sinc-function signal. The waveform function of the 
transmitted Sinc signal is $\sin x / x$. The frequency, Sinc length and sample rate of the Sinc signal are defined as $1 \mathrm{MHz}, 6000$, and $1 \mathrm{GSa} / \mathrm{s}$, respectively. There always exists jitter on received signal due to noise, so we take the average of 16 received signals as the final received signal. During the measurement, all setup devices are fixed except the LED samples. The difference of the amplitude, rise-time, fall time and overall signal shape of the received signal for three LED samples can feedback the communication performance difference of Si-substrate LEDs with different SL period number. The one with the shortest signal delay always has the widest bandwidth and shortest carrier lifetime if LED samples have the identical active area to make sure the similar resistancecapacitance (RC) constant. The one with highest amplitude of the received signal and most similar waveform shape with the transmitted signal is expected to have the highest SNR. In addition, two cases including small-signal response and large-signal response cases are investigated in this work. In the small-signal response case, the peakto-peak voltage (Vpp) of the Sinc-signal is set to $0.6 \mathrm{~V}$, and the transient response mainly shows the linear distortion resistance and response speed of LED. The nonlinear resistance of LED could be indicated from the large-signal response case where the $\mathrm{Vpp}$ is set to $1.2 \mathrm{~V}$.

\section{Results and discussion}

The optoelectronic characteristics of the three LED samples are first studied and presented in Fig. 3. The open voltage of three samples is different according to the results in Fig. 3a). Sample A has the highest open voltage of $3.3 \mathrm{~V}$, but that of Sample B and C are only around $2.5 \mathrm{~V}$. When injection current is identical, the operation voltage of Sample A is higher than that of Sample B, which is higher than Sample C. The variation trend of operation voltage indicates that the increase of SL period number effectively decreases the overall resistance and the operation voltage of Si-substrate LEDs. Figure 3b) depicts the output optical power under various current densities for three LED samples. Sample C has the highest optical power than Sample B and Sample A, showing that more SL period number could increase the EQE to some extent. The maximum output optical power of Sample B and C current has exceeded $1 \mathrm{~W}$ at the density over $70 \mathrm{~A} / \mathrm{cm}^{2}$. Besides, the linear power response curve could partially prevent the undesired nonlinear effect generated during the electro-optical conversion process, which improves the available SNR of VLC system. In a typical indoor VLC system, the required injection current needs to be higher than $10 \mathrm{~mA}\left(1 \mathrm{~A} / \mathrm{cm}^{2}\right.$ for $\left.45 \mathrm{mil} \mathrm{LED}\right)$ for an efficient communication. In this case, the Wall-plug efficiency of Sample C could approach 70\% according to the measurement results in Fig. 3c). Sample A with the fewest SL period numbers only have the highest WPE of $28 \%$, which is much lower than that of Sample B and C. The reason why more SL period number can improve the lighting performance of Si-substrate LEDs has been reported in Ref. [19]. It is the growth of V-shaped pits as the increase of SL period number that contributes to this improvement. The V-shaped pits are the inverted hexagonal pits on InGaN MQW structures triggered by threading dislocation. The recombination of carriers and holes is more likely to happen at the side wall of $\mathrm{V}$-shape pits than at the normal flattenregion of MQWs, and thus the larger V-pit facilitates the hole injection which causes the increase of $\mathrm{EQE}$ and the decrease of operation voltage. Finally, the spectrum characteristics of three samples are given in Fig. 3d), consisting of the peak wavelength and 

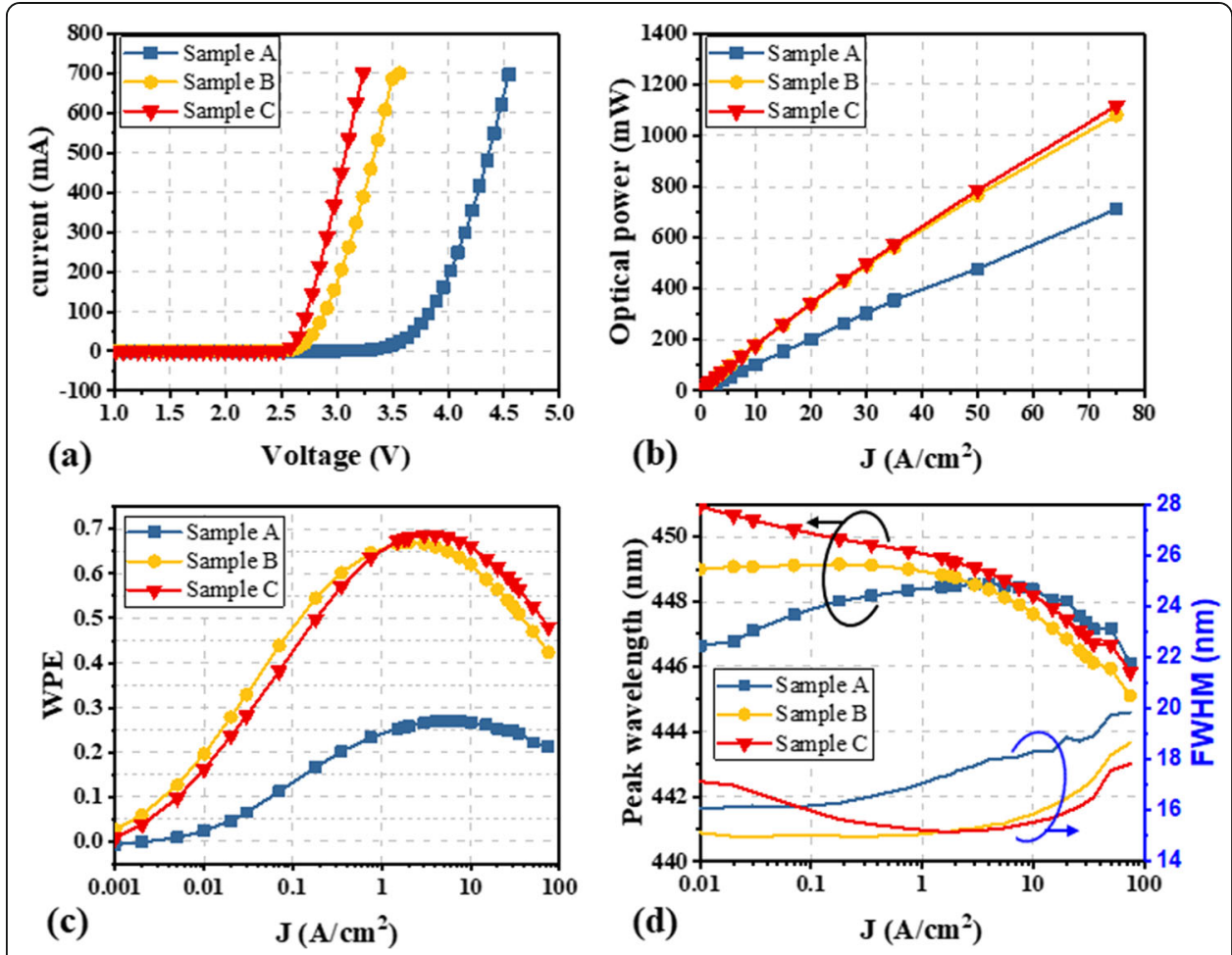

Fig. 3 Optoelectronic characteristics of LED Samples A, B, and C. a Current-voltage (I-V) characteristics under a CW injection current. b. Output optical power; c Wall-plug efficiency (WPE); d Peak wavelength and full width at half maximum (FWHM) of Samples A, B, and C under various current density (J)

the full width at half maximum (FWHM). There is a red shift and a subsequent blue shift for Sample A when current density moves from $0.01 \mathrm{~A} / \mathrm{cm}^{2}$ to $100 \mathrm{~A} / \mathrm{cm}^{2}$. Nevertheless, there is only a blue shift for Sample B and Sample C as the increase of current density. Overall peak wavelength for three samples varies between $446 \mathrm{~nm}$ to $455 \mathrm{~nm}$. The FWHM of three samples are lower than $20 \mathrm{~nm}$. Sample C has the widest FWHM when current density is lower than $0.1 \mathrm{~A} / \mathrm{cm}^{2}$, but has the narrowest FWHM when current density is higher than $8 \mathrm{~A} / \mathrm{cm}^{2}$.

To systematically evaluate the VLC performance of LED samples, there are four aspects of the device should be measured, including the frequency response, timedomain transient response, SNR and the maximum achievable data rate. The frequency response could reflect the bandwidth of a LED, and the transient response at the time domain could help analyze the distortion on waveform induced by the LED. The SNR and achievable data rate finally determine the communication capacity of the VLC system using this LED sample.

Figure 4 shows the S21 parameters of three LED samples. When the current is only $50 \mathrm{~mA}$, Sample C has the largest - 10-dB bandwidth of $220 \mathrm{MHz}$, but Sample B and A have the $-10 \mathrm{~dB}$ bandwidth of $201 \mathrm{MHz}$ and $57 \mathrm{MHz}$. Since more SL could relieve more stress inside MQWs, carrier recombination becomes easier when SL period number rises from 8 to 15 , and hence the carrier lifetime accordingly decreases, which is also presented from the great bandwidth extension of Sample B compared to Sample A. However, 15 SLs has already relieved most of the stress and realizes a good crystal quality, and thus the bandwidth of 32 SLs barely increases compared to that of 15 SLs. 

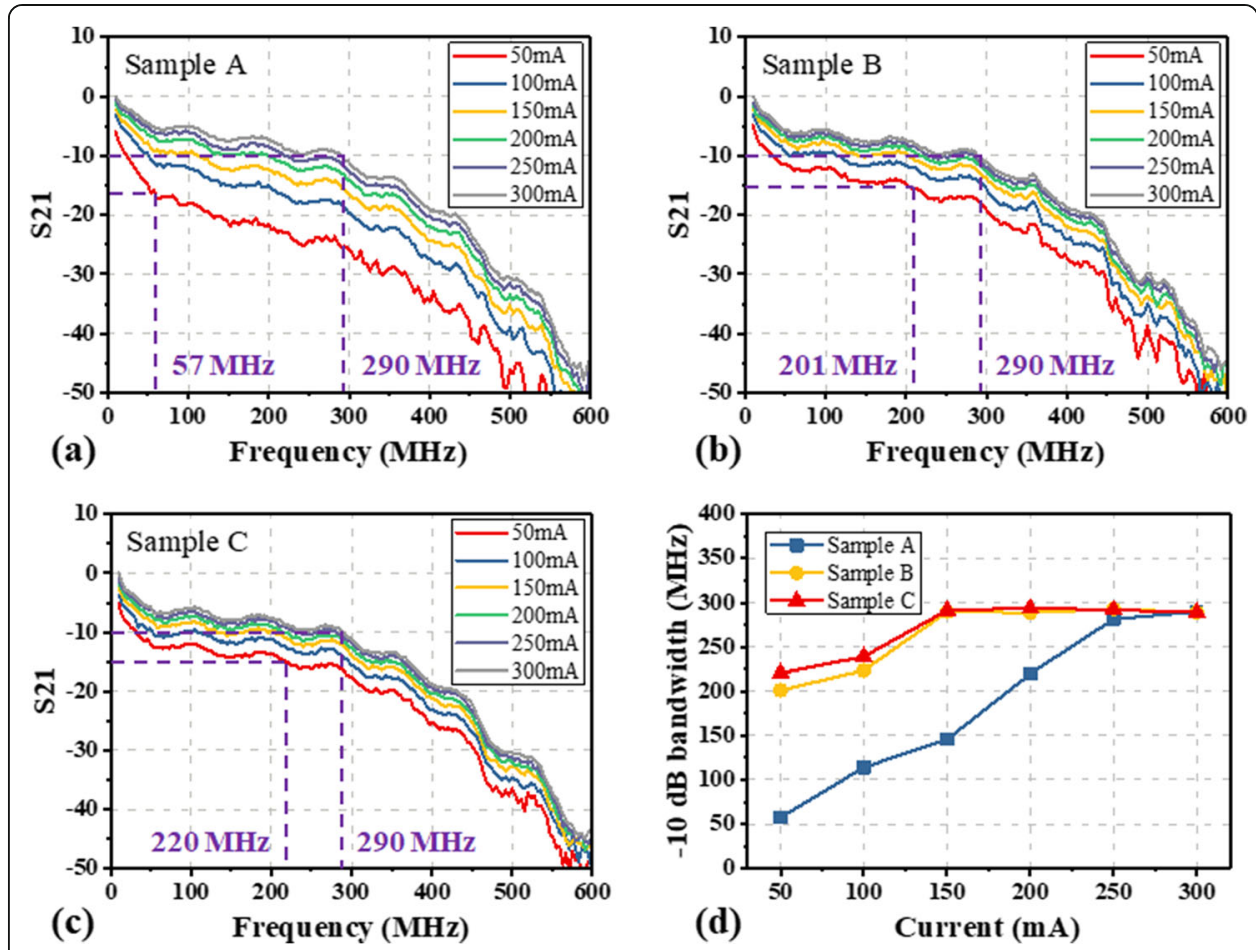

Fig. 4 a-c S21 parameters of Sample A-C under different injection current from $50 \mathrm{~mA}$ to $300 \mathrm{~mA}$. Their 10-dB bandwidth values at $50 \mathrm{~mA}$ and $300 \mathrm{~mA}$ are marked. $\mathbf{d}$ The $-10-\mathrm{dB}$ bandwidth of three samples under different current

As the injection current increases to $300 \mathrm{~mA}$, the $-10-\mathrm{dB}$ bandwidth of three samples all rise, finally to a steadily and identical level of $290 \mathrm{MHz}$. At such a high injection current, the dominant limitation for the increase of bandwidth has been changed from carrier lifetime to the RC constant, of which three samples are the same due to their identical LED size.

Next, the SNR performance of the VLC systems with Sample A-C is compared in Fig. 5. The baud rate and Vpp of the training signal for SNR estimation is set to 550 MBaud and $0.6 \mathrm{~V}$. At the current of $50 \mathrm{~mA}$, there is up to $1.6 \mathrm{~dB}$ SNR gain for Sample C compared to Sample B, and 5 dB SNR gain for Sample B compared to Sample A, which means that more SL period number would bring higher overall SNR response, benefiting the achievement of a higher data rate. However, due to the influence of the ND filter, the SNR of Sample C, which is the highest at the current of $50 \mathrm{~mA}$, inversely decreases the fastest as the increase of current compared to that of other samples. At the current of $300 \mathrm{~mA}$, the SNR of Sample C is the lowest. Sample A has the highest SNR in this case. This impact of the ND filter on SNR's decrease will be studied in the theoretical derivation section (Fig. 8).

To further demonstrate the positive impact of the increase of SL period number on communication quality, we subsequently investigate the time-domain transient response of three LED samples under small- and large-signal cases. The waveform of the received signal in the small-signal case is shown in Fig. 6a-c). Three samples have similar rise time among different current but have an obvious difference in fall time. Sample A has the longest time delay, and a bigger signal "delay tail" exists at the signal falling edge. In contrast, the amplitude of delay tail of Sample B and C is smaller than that of 

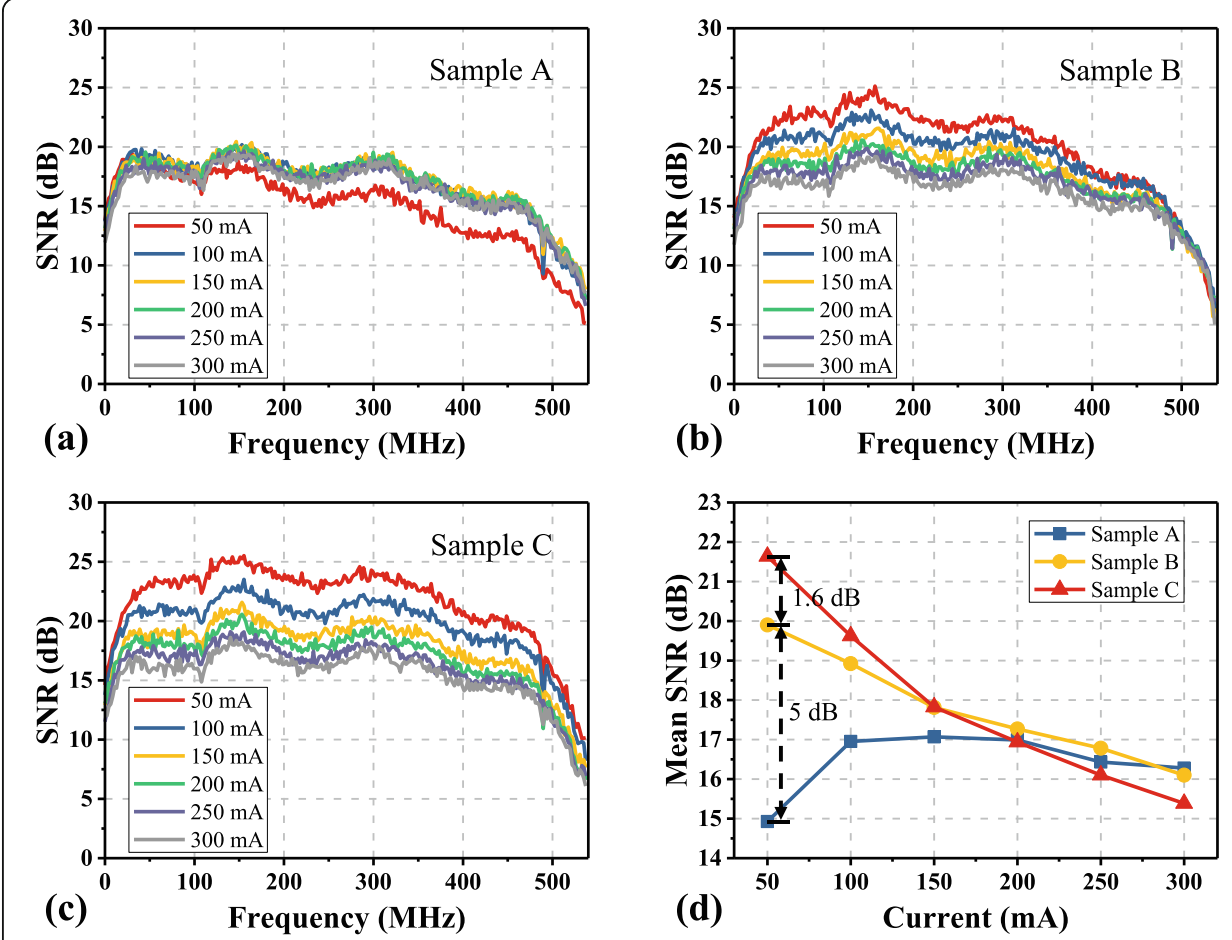

Fig. 5 a-c Estimated SNR versus frequency of Sample A-C under different injection current from $50 \mathrm{~mA}$ to $300 \mathrm{~mA}$. d The mean SNR of three samples under different current

Sample A, indicating Sample B and C have faster signal responses, i.e. wider bandwidths, than Sample A. Sample B has a similar bandwidth as Sample C at the smallsignal case owning to their similar amplitude of delay tail. The obtained bandwidth trend is consistent with the measured results in Fig. 4. Subsequently, the amplitude of the Sinc signal is increased to $1.2 \mathrm{~V}$ to study the large-signal response. From the results in Fig. 6d-f), the increase of amplitude greatly lifts the amplitude and duration of the delay tail, which will cause harmful inter-symbol-interference (ISI) and nonlinear effect on signal transmission. Sample $C$ has the smallest "delay tail", which suggests a more superior response performance than Sample B, of which the response performance is better than that of Sample A.

The amplitude response results (Fig. 7) suggest that more SL period number will improve the optical signal response ability of LED, outputting an optical signal with higher amplitude if the loaded electrical signal with a fixed Vpp in both the small- and large signal cases. When Vpp is $0.6 \mathrm{~V}$, the mean signal amplitude of Sample $\mathrm{C}$ is the highest, followed by Sample B and Sample C. This amplitude difference is consistent with the spectral efficiency (SE) difference, as well as the SNR difference in Fig. 5 implying that SNR and SE that a LED can achieve substantially are determined by the received signal amplitude. The higher the amplitude of received signal is, the higher SE and SNR are. The ND filter causes a faster attenuation to the amplitude of the LED with a higher amplitude response when the current increases, which is also found in the results of SNR measurement. At the high Vpp case (1.2 V) in Fig. 7b), Sample C still has the highest amplitude response, but a serious nonlinear effect suppresses the increase of amplitude, and thus the amplitude difference between Sample B and C 

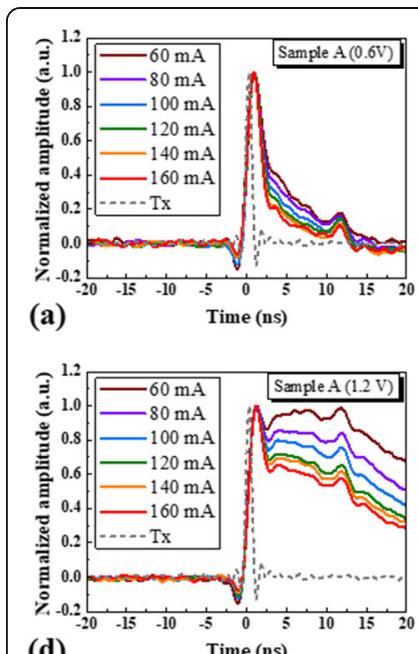

(d)

Time (ns)
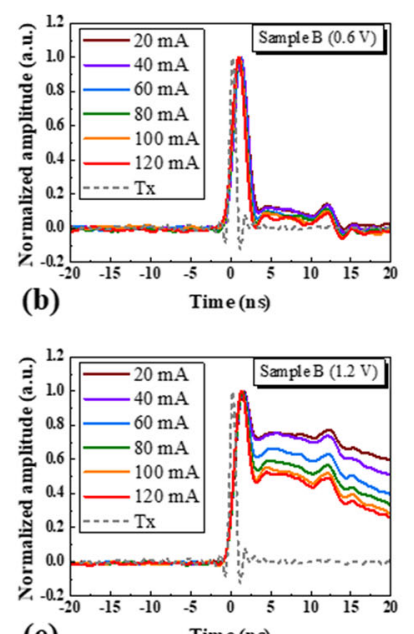

(e)
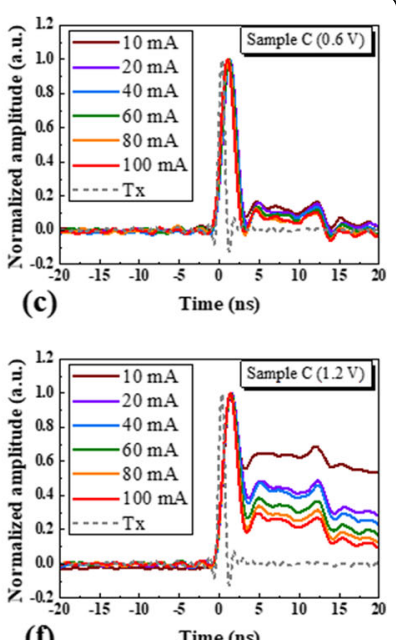

(f)

Fig. 6 The electrical signal response of three LED samples at different bias currents when injecting a Sincshaped pulse signal (a-c) with the amplitude of $0.6 \mathrm{~V}$, or $(\mathbf{d}-\mathbf{f})$ with the amplitude of $1.2 \mathrm{~V}$

becomes smaller than the small-signal case. Additionally, the SE at the low current range from 10 to $40 \mathrm{~mA}$ is lower than that of the small-signal case because the signal with 1.2 Vpp sustains more serious signal clipping at such a low bias current. Once the bias is high enough, signal clipping effect disappears, SE will accordingly rise.

Overall, the increase of SL period number could increase the amplitude of received signal, accelerate the signal response and greatly suppress the amplitude of signal delay tail to resist the underlying linear and nonlinear distortion in VLC system. However, how the increase of SL period number could provide this enhancement on signal quality is still unclear. In addition, the phenomenon that the system SNR for the LED with 32 SLs drops faster than the LED with 8 and 15 SLs also needs an explanation. Hence, a theoretical VLC model is established to explain these questions through the analysis of the electron-hole recombination mechanism in LED and signal modulation process.

The single-LED VLC system model is illustrated in Fig. 8a) with three parts included: transmitter, receiver and channel. The transmitter consists of a drive circuit module and a LED to convert the electrical voltage signal $(V(t))$ into a driving signal $\left(V(t)+V_{c}(t)\right)$, which is then transferred to an optical signal $(O(t))$ with average
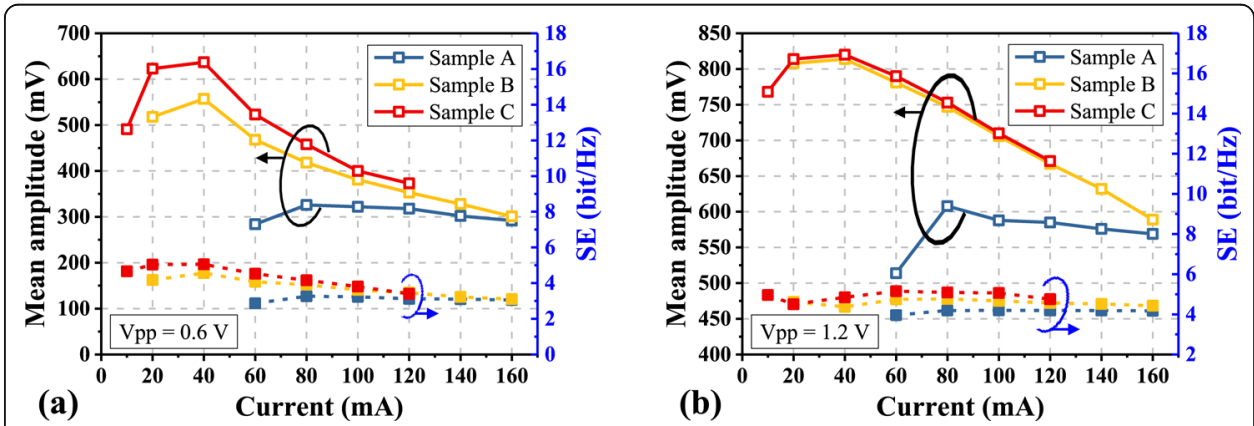

Fig. 7 The mean amplitude and SE of the received signal versus bias current when $\mathbf{a} \mathrm{Vpp}$ is $0.6 \mathrm{~V}$ and $\mathbf{b}$ Vpp is $1.2 \mathrm{~V}$. The baud rate of train signal is $550 \mathrm{MBaud}$ 

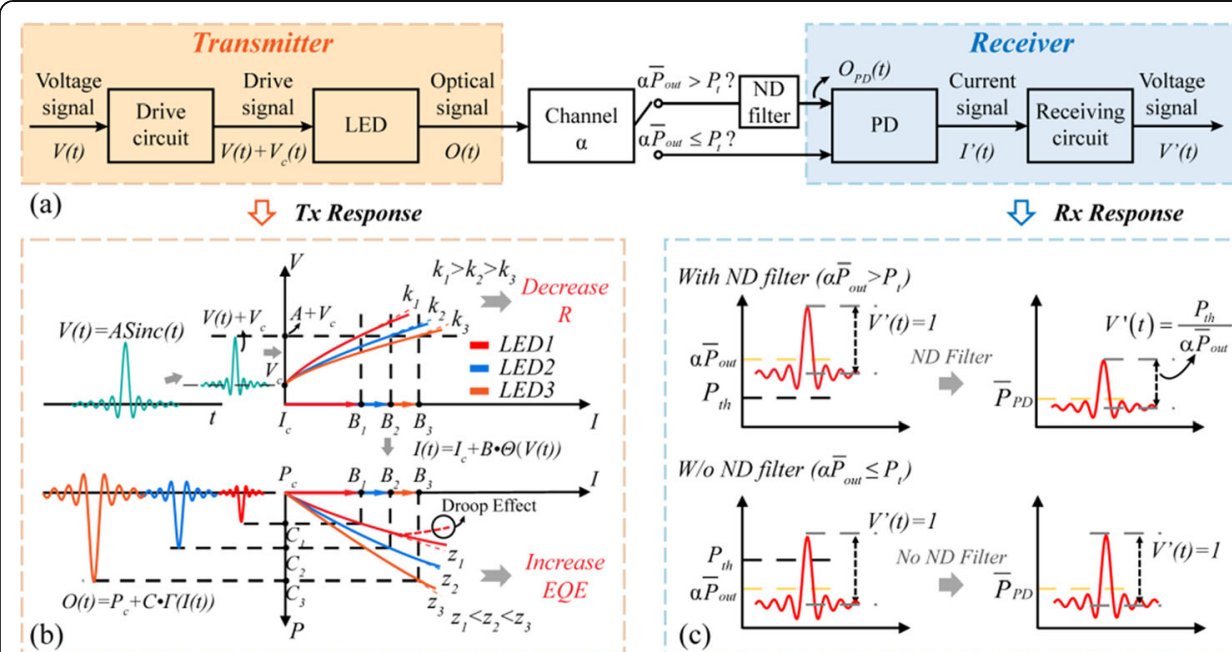

Fig. 8 Schematic illustration of a VLC system model. a Schematic diagram of a typical VLC system. $\mathbf{b}$ Illustration for the signal response for LEDs with different optoelectronic characteristics. $\mathbf{c}$ Impact on signal response when the neutral density (ND) filter is applied or not applied. The baud rate of train signal is 550 MBaud

transmit optical power $\left(\bar{P}_{\text {out }}\right)$. The $V_{c}(t)$ is a bias direct voltage. After the light passes the free-space channel, the received optical power is usually derived by multiplying $\bar{P}_{\text {out }}$ and the path loss $\alpha$ on a linear scale [30]. If the received optical power $\alpha \bar{P}_{\text {out }}$ is lower than the threshold power of photodetector (PD) saturation effect $\left(P_{t}\right)$, which is the maximum optical power that the utilized PD can respond, the PD can directly convert the received optical signal $\left(O_{P D}(t)\right)$ into an electrical current signal $\left(I^{\prime}(t)\right)$. Then, receiver circuit modules amplify and convert $I^{\prime}(t)$ into a voltage signal $\left(V^{\prime}(t)\right)$. If $\alpha \bar{P}_{\text {out }}$ is higher than $P_{t}$, there usually exists an ND filter before PD to manually attenuate $\alpha \bar{P}_{\text {out }}$ to $P_{t}$. However, the use of an ND filter also decreases the amplitude of the received optical signal besides the direct optical power, which is illustrated in Fig. 8 (c). The attenuation ratio should be $P_{\text {th }} /\left(\alpha \bar{P}_{\text {out }}\right)$ to reduce $\alpha \bar{P}_{\text {out }}$ to $P_{t}$. After the attenuation by ND filter, the output optical power is converted from the $\alpha \bar{P}_{\text {out }}=P_{D C}+P_{A C}$ to $O_{P D}=\frac{P_{t h}}{\alpha \bar{P}_{\text {out }}} P_{D C}+\frac{P_{\text {th }}}{\alpha \bar{P}_{\text {out }}}$ $P_{A C}$. The $P_{D C}$ is the direct optical power. The $P_{A C}$ is the modulated optical signal power that has been attenuated to $\frac{P_{\text {th }}}{\alpha \bar{P}_{\text {out }}} P_{A C}$. The calculated power loss and decay speed are $\left(1-\frac{P_{t h}}{\alpha \bar{P}_{\text {out }}}\right) P_{A C}$ and $\left(1-\frac{P_{\text {th }}}{\alpha \bar{P}_{\text {out }}}\right)$, respectively. The amplitude of the electrical received signal is proportional to the optical signal power. Assuming received electrical signal $V^{\prime}(t)$ has the amplitude of 1 without the ND filter's existence, the amplitude of $V^{\prime}(t)$ when adding a ND filter becomes $P_{\text {th }} /\left(\alpha \bar{P}_{\text {out }}\right)$. When injection current gradually grows, the $\alpha \bar{P}_{\text {out }}$ increases and thus the decay speed becomes faster. The expression of decay speed implies that higher direct optical power leads to a faster decay speed of the optical signal. Therefore, the signal amplitude of Sample C drops faster than other LED samples due to its highest WPE causing the highest output optical power at the same injection current. 
To obtain a higher system SNR equals to increase the amplitude $O(t)$ by optimizing the transmitter or increase the amplitude $I^{\prime}(t)$ by optimizing the receiver, meanwhile guaranteeing the noise power stays at a similar or lower level. Herein, we mainly discuss the optimization of the LED design to increase the amplitude $O(t)$. Figure 8 (b) is the schematic diagram of signal response during the LED's intensity modulation process. Provided a Sinc-function electrical signal $(V(t)=A \operatorname{Sinc}(t)=A \sin (t) / t)$ to the transmitter, the excited current signal inside the LED can be written as $I(t)=I_{c}(t)+B$ . $\Theta(V(t))$. Former $\left(I_{c}(t)\right)$ and later $(B \cdot \Theta(V(t)))$ refer to a DC and AC component, respectively. The $B$ and $\Theta(\cdot)$ stand for the amplitude of the current signal and the nonlinear response of V-I response, respectively. According to the Shockley equation, the comprehensive V-I transfer characteristic of a forward-biased $p-n$ junction is given by:

$$
I(t)=\frac{V(t)-I(t) R_{s}}{R_{p}}+I_{s} \exp \left(\frac{q\left(V-I R_{s}\right)}{n_{\text {ideal }} k T}\right)
$$

the series parasitic resistance $R_{s}$ and the parallel parasitic resistance $R_{p}$ frequently appear in LEDs caused by excessive contact resistance, neutral regions resistance and any channel that bypass the p-n junction. The $n_{\text {ideal }}, q, k, T$ are the ideality factor of the diode, electron charge, Boltzmann constant and absolute temperature, respectively. The $I_{s}$ is reverse saturation current, related to electron and hole diffusion constants, etc. The specific explanation can be learned in Ref. [31].

From the illustration of the V-I curve in Fig. 8b), the LED with a lower slope of V-I curve for any bias voltage point $\left(k_{3}<k_{2}<k_{1}\right)$ can derive a bigger value of signal amplitude $\left(B_{3}>B_{2}>B_{1}\right)$. The slope of V-I curve $(k)$ is provided by:

$$
k=\frac{d V}{d I}=R_{s}+\frac{1}{\frac{1}{R_{p}}+\frac{I_{s} q}{n_{\text {ideal }} k T} \exp \left(\frac{q\left(V-I R_{s}\right)}{n_{\text {ideal }} k T}\right)}
$$

Based on the analysis in Supplementary Section, decreasing $R_{s}$ or $R_{p}$ could decrease the slope and obtain a higher current signal response.

After the V-I response, the injection current brings the excess carrier concentrations of electrons $n_{e}(t)$ and holes $p_{e}(t)$ in the quantum well $(\mathrm{QW})$ region. The AC component causes the intensive change of the concentration of the injected carriers. These injected carriers are created and recombined in pairs within the LED either as radiative recombination, outputting an optical signal, or as non-radiative recombination. The variation of carrier concentration leads to the variation of the power of output light which stands for the modulated optical signal. The relationship of optical power $P(t)$ and carrier concentration is given in Ref. [32]:

$$
P(t)=w A \overline{E_{p}} B \cdot \eta_{E Q E} \cdot n_{e}(t)\left[n_{e}(t)+p_{0}\right]
$$

Where $w$ and $A$ represent the thickness and area of the active layer, $\overline{E_{p}}$ is the average photon energy. The radiative recombination constant $B$ depends on the carrier concentration and temperature. The $\eta_{E Q E}$ is the external quantum efficiency. The $p_{0}$ is the doping concentration. It's observed the output optical signal explicitly has a nonlinear relation with the injection current. Here, the output optical signal can be denoted as $O(t)=P_{c}+C \cdot \Gamma(I(t))$, including the DC optical power $P_{c}$ and the AC component $C$ $\cdot \Gamma(I(t))$. The $C$ and $\Gamma(\cdot)$ respectively stand for the amplitude of the optical signal and 
the nonlinear response of I-P response. However, Eq. (3) is only suitable for constant current or small-amplitude signal. For large amplitude signal where the peak is close to the bias current, the $\eta_{E Q E}$ will vary with the $A C$ signal. Therefore, the amplitude $C$ for a sampled symbol from time slot $t_{1}$ to $t_{2}$ can be calculated by:

$$
C=P\left(t_{2}\right)-P\left(t_{1}\right)=w A \overline{E_{p}} B \cdot\left[\xi\left(t_{2}\right)-\xi\left(t_{1}\right)\right]
$$

with

$$
\xi(t)=\eta_{E Q E}(t) \cdot n_{e}(t)\left[n_{e}(t)+p_{0}\right]
$$

From the above equations, the best approaches to increase value $C$ first is improving the overall external quantum efficiency (EQE) at every injection current, second, is mitigating the droop effect and maintaining the EQE still at a high level under a high current density state. In the illustration of the I-P curve in Fig. 8b), this increase of $\mathrm{EQE}$ helps increasing the curve slope, and get a relatively higher value of $\eta_{E Q E}\left(t_{1}\right)$, $\eta_{E Q E}\left(t_{2}\right)$, thereby the amplitude response $(C)$ can be higher. However, EQE normally decreases with the increase of injection current due to the droop effect [33]. If the droop effect is too serious, the output power of a LED even decreases at high current density, illuminated in Fig. 8b). The induced nonlinearity of I-P response also significantly damages the response of $C$ and SNR. Hence, increasing the EQE at both low and high current density by droop effect mitigation or other methods could benefit the modulation depth of LED, increasing the VLC system's SNR.

The method of reducing overall resistance (i.e. forward voltage) and increasing the EQE of LED could be unified into the enhancement of WPE of LED according to its concept [34]:

$$
W P E=E Q E \times \frac{h v}{q V}
$$

Where $h v, q, V$ are photon energy, elementary charge, the operation voltage of LEDs, respectively. Sample $C$ with the highest WPE is exactly the one with the highest SNR when the current density is higher than $1 \mathrm{~A} / \mathrm{cm}^{2}$. In conclusion, The LED with more SL period number presents a higher WPE at a relatively high injection current density, thereby provides a better optical signal response and higher SNR for VLC system. However, it's noted the conclusion doesn't mean the period number of SL should increase infinitively. Too many SLs would cause reverse function to VLC performance, i.e. the decrease of WPE and modulation efficiency. This is because the thickness of SLs is positively related to the series resistance of LED. When the SL period number is too large, the thickness will become the dominant factor to influence the overall resistance compared to the hole injection effect of a larger V-pit. In this case, the SL period number is supposed to be decreased to achieve a better VLC performance. Hence, there must exist an optimal SL period number to achieve the highest WPE. The optimal SNR response could be achieved using this SL period number.

BPL-DMT modulation is a practical bit allocation technology to utilize available SNR to maximum the SE of the VLC system. Figure 9 is the measured SE for three samples using the BPL-DMT modulation over a $1.2 \mathrm{~m}$ VLC link. The bias current and the Vpp of the transmitted signal are simultaneously adjusted to find the highest SE (i.e. the best working statue). Sample C has the highest SE compared to Sample B and A thanks to 


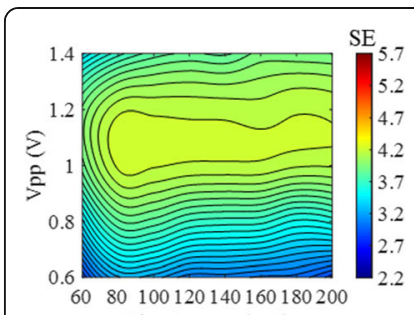

(a) Bias Current (mA)

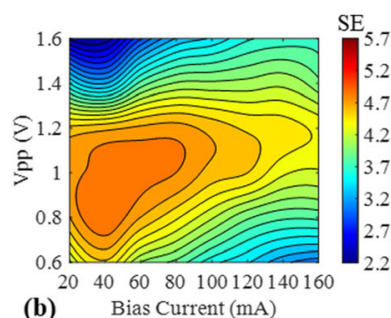

(b) Bias Current (mA)

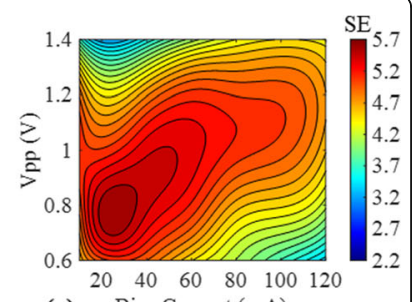

(c) Bias Current $(\mathrm{mA})$

Fig. 9 Measured average spectral efficiency (SE) of a Sample A, b Sample B, c Sample C at different peakto-peak voltage (Vpp) and bias current at a $1.2 \mathrm{~m}$ VLC system

the optimization of SL period number, so a high-speed VLC link is expected to be realized using Sample C and BPL-DMT modulation. Meanwhile, considering the indoor illumination distance for regular LED is within $3 \mathrm{~m}$, the highest data rate of VLC system at different transmission distances is studied using Sample C.

When transmission distance is extended to a $3 \mathrm{~m}$, the biggest challenge for the achieve of high data rate is the insufficient received optical power for receiver, where the noise of PD will submerge most of the signal, which causes a serious SNR loss. Therefore, the optimal current for the $1.2 \mathrm{~m}, 2 \mathrm{~m}$ and $3 \mathrm{~m}$ VLC link gradually rises from $20 \mathrm{~mA}$ to $80 \mathrm{~mA}$ shown in Fig. 10a). The best Vpp stays the level between $0.8 \mathrm{~V}$ and 1 V. A higher Vpp will lead to the nonlinear effect at the LED and a lower Vpp cannot provide enough SNR for high data rate transmission. Under the optimal current and Vpp, the VLC system using Sample C achieves the highest data rate of 3.37 Gbps, 3.24

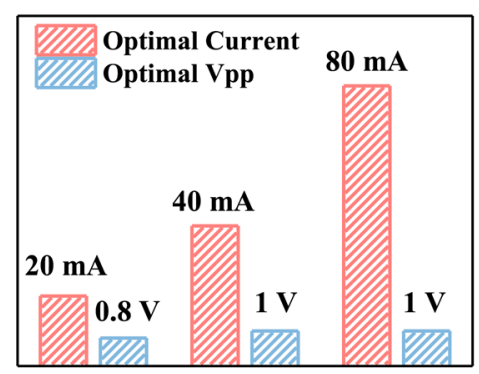

(a) $1.2 \mathrm{~m}$
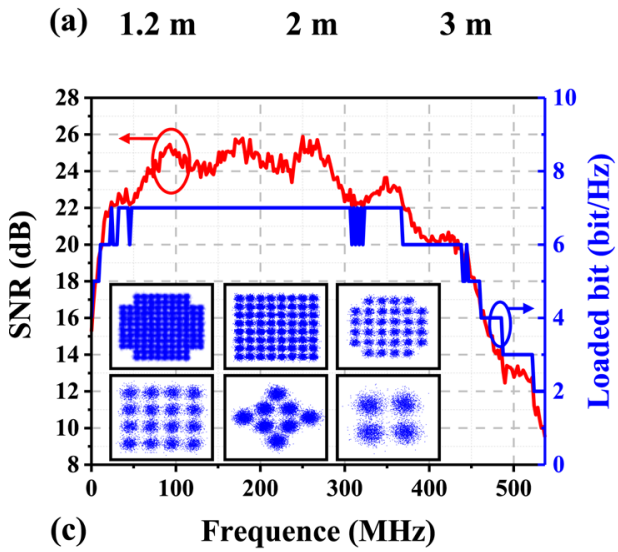
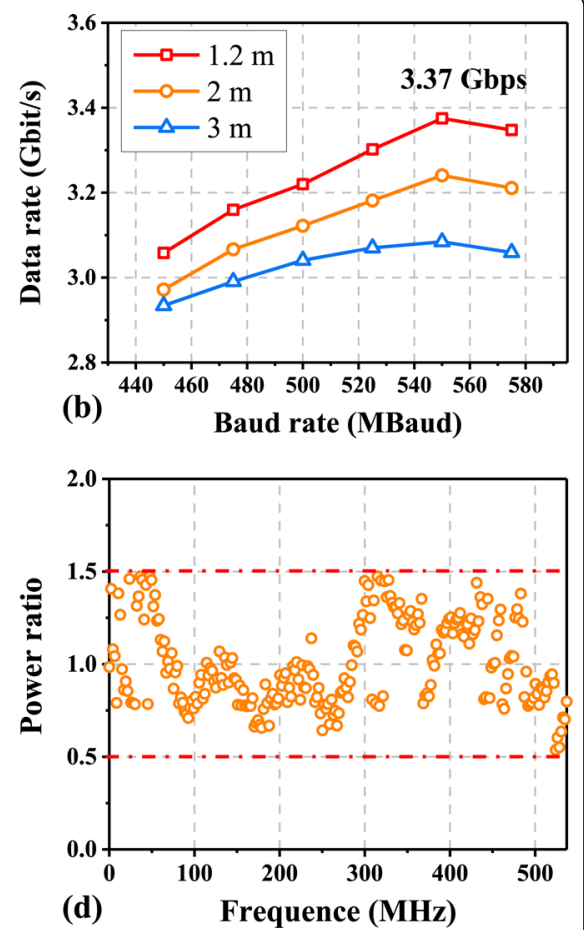

Fig. 10 a Optimal current and Vpp for Sample C to obtain the highest SNR. $\mathbf{b}$ Data rate versus different signal baud rate at the distance of $1.2 \mathrm{~m}, 2 \mathrm{~m}$ and $3 \mathrm{~m}$. c SNR and bit distribution at the highest data rate of 3.37 Gbps. Insets: allocated QAM signal diagrams with the loaded bit number from 2 bits/symbol to 7 bits/ symbol. d Power ratio at each DMT subcarrier after the bit and power loading technology 
Gbps and 3.08 Gbps respectively for $1.2 \mathrm{~m}, 2 \mathrm{~m}$ and $3 \mathrm{~m}$ at the baud rate of $550 \mathrm{MBaud}$ (Fig. 10b). All BERs is lower than the 7\% FEC threshold. To observe the bit and power allocation situation of the DMT signal with the highest data rate, the SNR, loaded bit number and power ratio for every subcarrier of the DMT signal are given in Fig. 10cd). At the frequency range between $50 \mathrm{MHz}$ and $350 \mathrm{MHz}$, the high SNR could support up to 128-QAM signal transmission and the lowest QAM order is binary phase shift keying (BPSK) at the low- and high-frequency range, which means the bit has been adaptively allocated to the channel. To exploit the power budget, the power ratios of every subcarrier are varied between 0.5 and 1.5. To the best of our knowledge, the data rate of $3.37 \mathrm{Gbps}$ is the highest communication capacity for a $1.2 \mathrm{~m}$ free-space $\mathrm{Si}$ substrate LED-based VLC system. The data rate of $3 \mathrm{Gbps}$ at the distance of $3 \mathrm{~m}$ also demonstrate that the Si-substrate LED could support the of synergetic work of highspeed communication and effectively indoor illumination.

\section{Conclusions}

Gearing towards the optimization of Si-substrate LED design for a higher speed VLC system, in this paper we for the first time analyzed the impact of superlattice interlayer period number on the data rate performance of the VLC system based on the Sisubstrate LED. From the experimental results, relatively more SL period numbers could increase the EQE, decrease the operation voltage and extend the bandwidth. The higher SNR response and optical signal amplitude response are attributed to the improvement of WPE, which has been validated by theoretical derivation. Besides, the transient response reveals that relatively more SL period number not only reduces the descent time of the time domain pulse but also provides nonlinear effect resistance to some extent. Using the Si-substrate LED with the optimal SL period number of 32, a 3.37 Gbps VLC system over a $1.2 \mathrm{~m}$ free-space link is successfully achieved, meantime maintaining 3 Gbps within a $3 \mathrm{~m}$ distance. As we are aware, this is the highest data rate for a $\mathrm{Si}$ substrate LED-based VLC system thanks to the optimization of device parameters. These results set a further step for the development of Si-substrate LEDs design and optimization typically for high-speed VLC systems.

\footnotetext{
Abbreviations

VLC: Visible light communication; LED: Light-emitting diode; SLs: Superlattice interlayers; RF: Radio-frequency; InGaN: Indium gallium nitride; GaN: Gallium nitride; Si: Silicon; SiC: Silicon carbide; EQE: External quantum efficiency; AIN: Aluminized nitrogen; AIGaN: Aluminum gallium nitride; CCS-MOCVD: Close-coupled showerhead metal-organic chemical vapor deposition; LT-GaN: Low-temperature GaN; MQWs: Multiple quantum wells; QCSE: Quantum-confined Stark effect; EBL: Electron blocking layer; SEM: Scanning electron microscopy; WPE: Wall-plug efficiency; SNR: Signal-tonoise ratio; AWG: Arbitrary waveform generator; Pre-Eq.: Pre-equalizer; EA: Electronic amplifier; PIN-PD: PIN photodetector; TIA: Trans-impedance amplifier; OSC: Oscilloscope; RC: Resistance-capacitance; ND filter: Neutral-density filter; DMT: Discrete multi-tone modulation; ZF Equ: Zero-forcing equalization; ISFA: Intra-symbol frequency-domain averaging; LC algorithm: Levin-Campello (LC) algorithm; QAM: Quadrature Amplitude Modulation; BPSK: Binary Phase Shift Keying; QPSK: Quadrature Phase Shift Keying
}

\section{Supplementary Information}

The online version contains supplementary material available at https://doi.org/10.1186/s43074-021-00039-9.

Additional file 1. This supplementary information provides a detailed derivation of the V-I curve slope of LED and explains why decreasing the series parasitic resistance and parallel parasitic resistance equals decreasing the overall resistance $(R)$ of LED. 


\section{Authors' contributions}

F.C.H conceived and conducted the experiments, analyzed the data and wrote the paper. F.C.H and S.Q.C carried out the measurement/data analysis about LED characteristics. S.Q.C, J. H, J.L.Z and F.Y.J fabricated and prepared the LED samples. Y.Y.Z and X.L.Z contribute to the measurement of LED's carrier recombination lifetime. G.Q.L and P. Z contributed to the data rate measurement. F.C.H, J.W.Z, S.Q.C, C. S mainly contributed to writing of the first draft with input from all other authors. N. C conceived the idea and supervised the research project. Z.X.H and S.H.Y help reply comments from reviewers. All authors discussed the results. The authors read and approved the final manuscript.

\section{Funding}

This work was partially supported by the NSFC project (No.61925104, No.62031011), Peng Cheng Laboratory project (No.PCL2021A14) and Fudan University-CIOMP Joint Fund.

\section{Availability of data and materials}

The datasets used and/or analyzed during the current study are available from the corresponding author on reasonable request.

\section{Declarations}

\section{Competing interests}

The authors declare that they have no conflict of interest.

\section{Author details}

1Key Laboratory for Information Science of Electromagnetic Waves (MoE), Fudan University, Shanghai 200433, People's Republic of China. ${ }^{2}$ National Institute of LED on Silicon Substrate, Nanchang University, Nanchang 330096, People's Republic of China. ${ }^{3}$ State Key Laboratory of Precision Spectroscopy, East China Normal University, Shanghai 200241, People's Republic of China. ${ }^{4}$ State Key Laboratory of Optical Communication Technologies and Networks, China Information Communication Technologies Group Corporation, Wuhan 430000, People's Republic of China. ${ }^{5}$ Peng Cheng Laboratory, Shenzhen 518055, China.

Received: 29 April 2021 Accepted: 22 July 2021

Published online: 09 August 2021

\section{References}

1. Calvanese Strinati E, Barbarossa S, Gonzalez-Jimenez JL, Ktenas D, Cassiau N, Maret L, et al. 6G: the next frontier: from holographic messaging to artificial intelligence using subterahertz and visible light communication. IEEE Veh Technol Mag. 2019;14(3):42-50. https://doi.org/10.1109/MVT.2019.2921162.

2. Chi N, Zhou Y, Wei Y, Hu F. Visible light communication in 6G: advances, challenges, and prospects. IEEE Veh Technol Mag. 2020;15(4):93-102. https://doi.org/10.1109/MVT.2020.3017153.

3. Chi N, Zhou Y, Liang S, Wang F, Li J, Wang Y. Enabling Technologies for High-Speed Visible Light Communication Employing CAP modulation. J Lightwave Technol. 2018;36(2):510-8. https://doi.org/10.1109/JLT.2017.2783906.

4. Chi N, Haas H, Kavehrad M, Little TDC, Huang X. Visible light communications: demand factors, benefits and opportunities [guest editorial]. IEEE Wirel Commun. 2015;22(2):5-7. https://doi.org/10.1109/MWC.2015.7096278.

5. De Vries JP, Simić L, Achtzehn A, Petrova M, Mähönen P. The Wi-fi "congestion crisis": regulatory criteria for assessing spectrum congestion claims. Telecommun Policy. 2014;38(8-9):838-50. https://doi.org/10.1016/j.telpol.2014.06.005.

6. Wang Y, Wang Y, Chi N, Yu J, Shang H. Demonstration of $575-\mathrm{Mb} / \mathrm{s}$ downlink and $225-\mathrm{Mb} / \mathrm{s}$ uplink bi-directional SCMWDM visible light communication using RGB LED and phosphor-based LED. Opt Express. 2013;21(1):1203-8. https://doi. org/10.1364/OE.21.001203.

7. Huang X, Wang Z, Shi J, Wang Y, Chi N. 1.6 Gbit/s phosphorescent white LED based VLC transmission using a cascaded pre-equalization circuit and a differential outputs PIN receiver. Opt Express. 2015;23(17):22034-42. https://doi.org/10.13 64/OE.23.022034

8. Bian R, Tavakkolnia I, Haas H. 15.73 Gb/s visible light communication with off-the-shelf LEDs. J Lightwave Technol. 2019; 37(10):2418-24. https://doi.org/10.1109/JLT.2019.2906464.

9. Zhou Y, Zhu X, Hu F, Shi J, Wang F, Zou P, et al. Common-anode LED on a Si substrate for beyond 15 Gbit/s underwater visible light communication. Photonics Res. 2019;7(9):1019-29. https://doi.org/10.1364/PRJ.7.001019.

10. Shin $H$, Jeon $K$, Jang Y, Gang M, Choi M, Park W, et al. Comparison of the microstructural characterizations of GaN layers grown on Si (111) and on sapphire. J Korean Phys Soc. 2013;63(8):1621-4. https://doi.org/10.3938/jkps.63.1621.

11. Härle V, Hahn B, Lugauer H J, et al. GaN based LEDs and lasers on SiC[J]. Phys Status Solidi A. 2000;180(1):5-13

12. Xiong C, Jiang F, Fang W, Wang L, Liu H, Mo C. Different properties of GaN-based LED grown on Si (111) and transferred onto new substrate. Sci China Series E. 2006;49(3):313-21. https://doi.org/10.1007/s11431-006-0313-1.

13. Wong WS, Sands T, Cheung NW, Kneissl M, Bour DP, Mei P, et al. Fabrication of thin-film InGaN light-emitting diode membranes by laser lift-off. Appl Phys Lett. 1999;75(10):1360-2. https://doi.org/10.1063/1.124693.

14. Ryu HY, Jeon KS, Kang MG, Yuh HK, Choi YH, Lee JS. A comparative study of efficiency droop and internal electric field for InGaN blue lighting-emitting diodes on silicon and sapphire substrates. Sci Rep. 2017;7(1):44814. https://doi.org/10.1 038/srep44814.

15. Dadgar A, Poschenrieder M, Bläsing J, Fehse K, Diez A, Krost A. Thick, crack-free blue light-emitting diodes on Si (111) using low-temperature AIN interlayers andin situSixNy masking. Appl Phys Lett. 2002;80(20):3670-2. https://doi.org/10.1 063/1.1479455.

16. Cheng K, Leys M, Degroote S, van Daele B, Boeykens S, Derluyn J, et al. Flat GaN epitaxial layers grown on Si (111) by metalorganic vapor phase epitaxy using step-graded AIGaN intermediate layers. J Electron Mater. 2006;35(4):592-8 https://doi.org/10.1007/s11664-006-0105-1. 
17. Zamir S, Meyler B, Salzman J. Thermal microcrack distribution control in GaN layers on Si substrates by lateral confined epitaxy. Appl Phys Lett. 2001;78(3):288-90. https://doi.org/10.1063/1.1338968.

18. Liu J, Feng F, Zhou Y, Zhang J, Jiang F. Stability of Al/Ti/au contacts to N-polar n-GaN of GaN based vertical light emitting diode on silicon substrate. Appl Phys Lett. 2011;99(11):111112. https://doi.org/10.1063/1.3640229.

19. Quan ZJ, Liu JL, Fang F, Wang GX, Jiang FY. A new interpretation for performance improvement of high-efficiency vertical blue light-emitting diodes by InGaN/GaN superlattices. J Appl Phys. 2015;118(19):6.

20. Tao X, Liu J, Zhang J, Mo C, Xu L, Ding J, et al. Performance enhancement of yellow InGaN-based multiple-quantumwell light-emitting diodes grown on Si substrates by optimizing the InGaN/GaN superlattice interlayer. Opt Mater Express. 2018;8(5):1221-30. https://doi.org/10.1364/OME.8.001221.

21. Mo C, Fang W, Pu Y, Liu H, Jiang F. Growth and characterization of InGaN blue LED structure on Si (111) by MOCVD. J Cryst Growth. 2005;285(3):312-7. https://doi.org/10.1016/j.jcrysgro.2005.08.046.

22. Liu L, Wang L, Li D, Liu N, Li L, Cao W, et al. Influence of indium composition in the prestrained InGaN interlayer on the strain relaxation of InGaN/GaN multiple quantum wells in laser diode structures. J Appl Phys. 2011;109(7):073106. https://doi.org/10.1063/1.3569848.

23. Jiang F, Wang L, Fang W. Semiconductor light-emitting device and method for making same: U.S. Patent 7, 919, 784[P]. 2011.

24. Huang $X$, Shi J, Li J, Wang Y, Chi N. A Gb/s VLC transmission using hardware Preequalization circuit. IEEE Photon Technol Lett. 2015;27(18):1915-8. https://doi.org/10.1109/LPT.2015.2445781.

25. Inan B, Jeffrey Lee SC, Randel S, Neokosmidis I, Koonen AMJ, Walewski JW. Impact of LED nonlinearity on discrete multitone modulation. J Opt Commun Netw. 2009;1(5):439-51. https://doi.org/10.1364/JOCN.1.000439.

26. Shafik RA, Rahman MS, Islam AHMR, leee. On the extended relationships among EVM, BER and SNR as performance metrics. In: Icece 2006: Proceedings of the 4th International Conference on Electrical and Computer Engineering; 2006. p. 408-+.

27. Levin HE. A complete and optimal data allocation method for practical discrete multitone systems. In: GLOBECOM'01. IEEE Global Telecommunications Conference (Cat. No.01CH37270); 2001.

28. Campello J. Optimal discrete bit loading for multicarrier modulation systems. In: Proceedings. 1998 IEEE International Symposium on Information Theory (Cat. No.98CH36252); 1998.

29. Chen M, He J, Tang J, Chen L. Pilot-aided sampling frequency offset estimation and compensation using DSP technique in DD-OOFDM systems. Opt Fiber Technol. 2014;20(3):268-73. https://doi.org/10.1016/.yofte.2014.02.011.

30. Hussain B, Li X, Che F. Yue CP, and Wu L, visible light communication system design and link budget analysis. J Lightwave Technol. 2015;33(24):5201-9. https://doi.org/10.1109/JLT.2015.2499204.

31. Schubert EF, Gessmann T, Kim JK. Light emitting diodes. In: Kirk-Othmer Encyclopedia of Chemical Technology; 2000.

32. Windisch R, Knobloch A, Kuijk M, Rooman C, Dutta B, Kiesel P, et al. Large-signal-modulation of high-efficiency light-emitting diodes for optical communication. IEEE J Quantum Electron. 2000;36(12):1445-53. https://doi.org/10.1109/3.892565.

33. Piprek J. Efficiency droop in nitride-based light-emitting diodes. Phys Status Solidi (a). 2010;207(10):2217-25.

34. Kuritzky LY, Espenlaub AC, Yonkee BP, Pynn CD, DenBaars SP, Nakamura S, et al. High wall-plug efficiency blue III-nitride LEDs designed for low current density operation. Opt Express. 2017;25(24):30696-707. https://doi.org/10.1364/OE.25.030696.

\section{Publisher's Note}

Springer Nature remains neutral with regard to jurisdictional claims in published maps and institutional affiliations.

\section{Submit your manuscript to a SpringerOpen ${ }^{\circ}$ journal and benefit from:}

- Convenient online submission

- Rigorous peer review

- Open access: articles freely available online

High visibility within the field

- Retaining the copyright to your article

Submit your next manuscript at $>$ springeropen.com 